\title{
Microvascular invasion Risk Scores affect the estimation of early Recurrence after Resection in Patients with Hepatocellular Carcinoma: A Retrospective Study
}

\section{Sheng Wang}

Taizhou First People's Hospital

Weizhi Zheng

Taizhou First People's Hospital

Zhencheng Zhang

Taizhou First People's Hospital

\section{Guohua Zhang}

Taizhou First People's Hospital

Danjiang Huang ( $\square$ zjhyhdj@163.com )

Taizhou First People's Hospital

\section{Research Article}

Keywords: microvascular invasion, hepatocellular carcinoma, risk score, recurrence

Posted Date: February 10th, 2022

DOI: https://doi.org/10.21203/rs.3.rs-1298158/v1

License: (c) (i) This work is licensed under a Creative Commons Attribution 4.0 International License. Read Full License 


\section{Abstract}

Background: Microvascular invasion (MVI) is a histological factor that is closely related to the early recurrence of hepatocellular carcinoma (HCC) after resection. To investigate whether the preoperative risk factors based on MVI status can be used to estimate early recurrence of HCC after resection.

Methods: Between January 2018 to March 2021, a total of 108 patients with surgically treated single HCC was retrospectively included in our study. Fifty-one patients were pathologically confirmed with MVI and 57 patients were absent of MVI. Univariate and multivariate logistic regression analysis of preoperative laboratory and magnetic resonance imaging (MRI) features were used to screen noninvasive risk factors in association with MVI in HCC. A risk score based on the odds ratio (OR) values of MVI-related risk factors was calculated to estimate the early recurrence after resection of HCC.

Results: In multivariate logistic regression analysis, tumor size $>2 \mathrm{~cm}(\mathrm{P}=0.024, \mathrm{OR}=3.05,95 \% \mathrm{Cl}$ 1.1911.13), PIVKA- $\triangle>32 \mathrm{mAU} / \mathrm{ml}(\mathrm{P}=0.001, \mathrm{OR}=4.13,95 \% \mathrm{Cl}: 1.23-11.38)$, irregular tumor margin $(\mathrm{P}=0.018$, $O R=3.10,95 \% \mathrm{Cl}: 1.16-8.31)$ and $A D C<1007 \times 10^{-3} \mathrm{~mm}^{2} / \mathrm{s}(\mathrm{P}=0.035, \mathrm{OR}=2.27,95 \% \mathrm{Cl}: 1.14-7.71)$ were independent risk factors correlated to $\mathrm{MVI}$ in HCC. Risk scores of patients were calculated and were then categorized into high, moderate and low risk levels. In multivariate cox survival analysis, only high risk score of MVI was an independent risk factor of early recurrence ( $P=0.026, O R=1.86,95 \% \mathrm{Cl}: 1.08-3.22)$, with a sensitivity and specificity of $0.52,0.88$, respectively.

Conclusions: A risk score based on MVI status can help stratify patients in high risk of early recurrence after resection of HCC.

\section{Background}

Hepatocellular carcinoma (HCC) is the most common liver cancer worldwide and the recurrence rate at 5 years after surgery for eligible patients with HCC is as high as 70\% [1-3]. Early recurrence is currently considered as intrahepatic reoccurrence of primary HCC during the first two years after surgery and is associated with worse overall survival. The aggressiveness of several pathological factors of HCC, including low tumoral differentiation, microvascular invasion, is high risk for early recurrence after resection [3-5].

Microvascular invasion (MVI) is one of the most important histological features that is closely related to postoperative early recurrence of HCC [6]. The treatments decision making may be changed for HCC patients with MVI $[7,8]$. Though MVI can only be identified by histology, considerable efforts have been made to provide a noninvasive method to predict MVI status of HCC. Currently, a-fetoprotein (AFP) and Prothrombin induced by vitamin $\mathrm{K}$ absence-II (PIVKA-II) are important serum tumor markers in the detection of HCC [9-12]. The elevated serum level of PIVKA-II is associated with MVI and tumor recurrence [12]. The radiological methods including morphologic features and quantitative imaging parameters have been widely explored for predicting MVI status in HCC [13-18]. Some imaging features such as the tumor size, irregular shape, tumoral or peritumoral enhancement pattern, capsule appearance 
et al, are valuable characteristics of MVI. The further quantitative analyses of the radiomics or deep learning approaches are promising but technically complex in regarded as "black box". [19, 20]. Unfortunately, a reliable predictive model of $\mathrm{MVI}$ in consensus for estimating recurrence-free survival of patients after resection of HCC for an easier clinical use is still in lack.

Several previous studies have established a predictive model derived from MRI variables to directly estimate the risk of early recurrence after resection of HCC [21-24]. However, in these MR imaging-based models, the relationship between the risk factors and MVI was unclear, so these risk factors cannot replace the crucial role of pathologically determined MVI status for predicting early recurrence. Whether a predictive model of MVI can be used to predict postoperative early recurrence needs to be identified. We proposed that a simplified point scale can reflect the impact of each variable on MVI for patient risk stratification of early recurrence and thus benefit patients from therapeutic decision making.

In our study, we aimed to develop a noninvasive risk score based on MVI to establish a predictive model for prognostic stratification of early recurrence in HCC patients after resection.

\section{Materials And Methods}

\section{Study population}

This is a retrospective study with ethics committee approved by the local institutional review board (approval number 2020-KY002-01) and the requirement for written informed consent waived. Between January 2018 to March 2021, 143 patients in suspicious of having HCCs were consecutively included in our study, after reviewing the institutional radiological and histological database. Inclusion criteria: (1) pathological diagnosis of single HCC after surgery with MVI status; (2) having preoperative MRI and laboratory tests no more than 2 weeks before surgery; (3) receiving no preoperative adjuvant treatments. 35 patients were excluded for: (1) 3 patients having two or multiple HCCs; (2) 6 patients having no preoperative MRI; (3) 15 patients having preoperative adjuvant TACE or targeted therapies; (4) 9 patients having been using relevant drugs (e.g. vitamin $\mathrm{K}$ and warfarin) that may affect the results of the PIVKA-II test; (5) 2 patients followed up for less than 6 months (Figure 1). Finally, a total of 108 patients with pathologically single $\mathrm{HCC}$ was included in our analysis, with 51 patients were presence of $\mathrm{MVI}$ and 57 were absence of MVI.

\section{Baseline characteristics and imaging variables}

The baseline characteristics of patients including age, gender, etiology, alanine aminotransferase (ALT), aspartate aminotransferase (AST), serum alpha-fetoprotein (AFP), PIVKA-II level and maximum tumor diameter were recorded. The PIVKA-II level was measured on an instrument (ARCHITECT i 2000SR) by using the same batch of PIVKA-II test reagents manufactured by the company (Abbott, USA). A PIVKA-II level $>32 \mathrm{mAU} / \mathrm{ml}$ is considered positive. 
Two experienced radiologists in consensus evaluated the following MR imaging features: (1) the maximum tumor diameter, measured on the pre-contrast phase during dynamic contrast-enhanced MRl; (2) tumor margin, categorized as smooth and irregular tumor margin; (3) presence or absence of capsule enhancement; (4) apparent diffusion coefficient (ADC) values, measured three times at each section of the whole tumor, and the average value of the three times was calculated.

\section{MRI protocol}

All MRI examinations were performed using a German Siemens 1.5 T magnetic resonance scanner with a body phased array coil. The standard imaging protocol included transverse T1-weighted fat suppression imaging (repetition time (TR):132.00ms, echo time (TE):5.09ms, bandwidth (BW):179.00, flip angle (FA): $60.00^{\circ}$, slice thickness: $6.00 \mathrm{~mm}$, layer spacing: $1.80 \mathrm{~mm}$ ), T2-weighted cross-sectional fat suppression imaging (TR:5500.18ms, TE:101.00ms, BW: 260.00, FA: $140.00^{\circ}$, slice thickness: $6.00 \mathrm{~mm}$, layer spacing:1.80mm), diffusion-weighted imaging ( $\mathrm{b}=800 \mathrm{~s} / \mathrm{mm} 2)$ and apparent diffusion coefficient (ADC, TR:5574.23ms, TE:72.00ms, BW:1736.00, FA: 90.00, slice thickness:6.00mm, layer spacing:1.80mm). The contrast agent gadopentetate was injected intravenously at a rate of $2 \mathrm{ml} / \mathrm{s}$ at 0.2 $\mathrm{mmol} / \mathrm{kg}$ and flushed with saline. Scans of arterial, portal and delayed phases were taken at $22 \mathrm{~s}, 60 \mathrm{~s}$ and 180 s post-injection, respectively.

\section{Pathological diagnosis of MVI}

The "7-point" baseline sampling method was used, with 1:1 sampling of tumor and peritumoral liver tissue at 12, 3, 6 and 9 points of the tumor [7]. The pathological diagnosis report included description of the gross specimen, immunohistochemical staining, and MVI status. MVI was diagnosed as presence of nesting clusters of tumor cells in the portal vein branches, hepatic vein or endothelium-lined vasculature under the microscope.

\section{Follow-up}

For HCC patients after resection, ultrasound and serum AFP test were performed every 3 months during the first 2 years and every 6 months thereafter during follow-up. All patients were follow-up for at least 6 months until the end of our study. Dynamic contrast-enhanced CT/MRI or ${ }^{18} \mathrm{~F}$-fluorodeoxyglucose positron emission tomography (PET-CT) would be performed for further evaluation if patients have suspicious recurrence detected on US or with elevated AFP during follow-up. The intrahepatic recurrence was identified by either pathological findings or typical imaging features of HCC $[25,26]$. Early recurrence was defined as intrahepatic recurrence during the first 2 years after resection of HCC [3].

\section{Statistical analysis}

Student's t-test was used for comparison between normally distributed variables that expressed as mean \pm standard deviation. Mann-Whitney $U$ test were used for comparison between non-normally distributed variables that expressed as median with range in parentheses. Chi-square test was used for comparison 
between categorical groups. Univariate and multivariate logistic regression was applied to screen the independent risk factors of $\mathrm{MVI}$ and early recurrence. Odds ratio (OR) with $95 \%$ confidence interval $(95 \% \mathrm{Cl})$ were calculated. The receiver operating characteristic $(\mathrm{ROC})$ curve of $A D C$ value with area under ROC curve (AUC) and the cutoff value calculated. Risk scores were simplified by rounding OR values of risk factors of MVI to nearest half for establishing a predictive model of early recurrence. The sensitivity, specificity, positive predictive value (PPV) and negative predictive value (NPV) of risk level of scores were calculated. $P$ value $<0.05$ was considered a statistically significant difference. SPSS software (version 21.0; SPSS, Chicago, III) was used for data analysis.

\section{Results}

\section{Patient characteristics}

A total of $108 \mathrm{HCC}$ patients (94 males and 14 females) were included in our study. Among the 108 patients, 51 patients were presence of $\mathrm{MVI}$ and 58 patients were absence of $\mathrm{MVI}$, with an average age of 61 years. The mean follow-up time of all $108 \mathrm{HCC}$ patients was $16.9 \pm 9.1$ months (range: 6-36 months). During follow-up, $46(42.6 \%)$ patients had early recurrence of HCC after resection, with an average time to early recurrence of $13.3 \pm 6.5$ months.

\section{Risk factors of MVI}

The demographic, laboratory and imaging characteristics were demonstrated in Table 1. The results showed that tumor diameter, serum PIVKA- level, tumor shape and ADC values were significantly different between MVI positive and negative groups. The AUC of ADC value for predicting MVI in $\mathrm{HCC}$ is 0.849 (0.776-0.922) with a cut-off value of $1007 \times 10^{-3} \mathrm{~mm}^{2} / \mathrm{s}$. In univariate analysis, the risk factor of tumor size $>2 \mathrm{~cm}, \mathrm{PIVKA}-\mathrm{X}>32 \mathrm{mAU} / \mathrm{ml}$, irregular shape, $\mathrm{ADC}<1007 \times 10^{-3} \mathrm{~mm}^{2}$ and capsule enhancement were associated with MVI in HCC patients (all $P<0.05)$. In multivariate analysis, tumor size $>2 \mathrm{~cm}(P=0.024$, $\mathrm{OR}=3.05,95 \% \mathrm{Cl}: 1.19-11.13), \mathrm{PIVKA}-\mathrm{\square}>32 \mathrm{mAU} / \mathrm{ml}(\mathrm{P}=0.001, \mathrm{OR}=4.13,95 \% \mathrm{Cl}: 1.23-11.38)$, irregular shape $(P=0.018, O R=3.10,95 \% \mathrm{Cl}: 1.16-8.31)$ and $A D C<1007 \times 10^{-3} \mathrm{~mm}^{2}(P=0.035, O R=2.27,95 \% \mathrm{Cl}: 1.14-$ 7.71) were independent risk factors of MVI. (Table 2) (Figure 2).

\section{Prognostic Stratification Based on Risk Score}

The risk score was weighted based on the OR values of MVI in multivariate analysis. The risk score of tumor size $>2 \mathrm{~cm}$, PIVKA- $\triangle>32 \mathrm{mAU} / \mathrm{ml}$, irregular tumor margin and $A D C<1007 \times 10^{-3} \mathrm{~mm}^{2} / \mathrm{s}$ was $3,4,3,2$ points, respectively. The risk factors of tumor size, PIVKA- $\triangle$ and $A D C<1007 \times 10^{-3} \mathrm{~mm}^{2} / \mathrm{s}$ were significantly different between patients with or without early recurrence while the irregular tumor shape showed no statistical significance. The risk score ranged from 0 to 12 points. Patients were then categorized as low risk (risk score: 0-4 points), moderate risk (risk score:5-7 points) and high risk (risk score: 8-12 points) groups. The high or low risk score level can significantly stratified prognostic difference of early recurrence. Among 108 patients of HCC, 36 patients had high risk score of MVI and 29 of them (29/36, \%) 
had early recurrence (Figure 2) (Table 3). In 49 patients with low risk score of MVI (Figure 3), 32 of them (32/49) did not had early recurrence. In univariate cox survival analysis, tumor size $>2 \mathrm{~cm}, \mathrm{PIVKA}-\mathbb{Z}>32$ $\mathrm{mAU} / \mathrm{ml}, \mathrm{ADC}<1007 \times 10^{-3} \mathrm{~mm}^{2}$ value, high or low risk score were statistically significant risk factors of early recurrence. In multivariate cox survival analysis, only high risk score of MVI was independent risk factor of early recurrence $(P=0.026, O R=1.86,95 \% \mathrm{Cl}: 1.08-3.22)$ (Table 4). The Kaplan-Meier curves in Figure 4 showed that the RFS in high risk groups of MVI was worse than moderate and low risk groups of MVI $(P=0.002)$. However, the RFS between moderate and low risk groups of MVI did not show significant difference.

\section{Diagnostic performance of risk score}

The sensitivity, specificity, PPV and NPV of high, moderate and low risk groups of MVI were shown in Table 5. The high risk group achieved a relative low sensitivity but high specificity of $0.52,0.88$ for estimating early recurrence. For low risk group, the sensitivity, specificity, PPV and NPV for estimating early recurrence was $0.60,0.69,0.65$ and 0.64 , respectively.

\section{Discussion}

Our study developed a simple risk score based on MVI status by evaluation of preoperative radiological and laboratory characteristics. A high risk score of MVI can provide a reliable prognostic stratification in early recurrence of HCC after resection.

In recent years, many scholars have paid attention to the noninvasive evaluation of MVI in HCC, because $\mathrm{MVI}$ is one of the most important pathological factors in association with aggressiveness of tumor and postoperative early recurrence.

Our study found that PIVKA-II-positive HCCs were more likely to have MVI than PIVKA-II-negative HCCs. PIVKA-II promotes proliferation of HCC cells and induces angiogenesis in surrounding liver tissue, thus promote vascular invasion of HCC [27, 28]. Previously, Yu et al [15] found that the sensitivity of PIVKA-II in diagnosing HCC was higher than that of AFP, especially in early-stage HCC. The elevated level of PIVKA-II tests was associated with MVI in $\operatorname{HCC}[9,12]$, which is in consistent with our findings. In our multivariate analysis, the PIVKA-II showed the highest OR value among the risk factors, indicating that PIVKA-II was the most important risk factor related to MVI in HCC.

Our risk score system contained some important imaging features in predicting MVI status of HCC. Consistent with previous reports, the larger tumor size and irregular tumor margin were proposed as significant risk factors that related to MVI in HCC. As the tumor size increases during progresses, the risk of MVI and intrahepatic metastasis increases. Pawlik et al [16] investigated the relationship between the tumor size in 1073 HCC patients and MVI, and found that single HCC larger than $5 \mathrm{~cm}$ had significantly increased incidences of MVI. The tumor margin evaluated on MRI reflects the pathological characteristics of gross appearance. The gross types of "nodular with extranodular growth" and "multinodular confluent type" showing irregular tumor margin on MRI may have a higher incidence of MVI in HCC [29, 30]. The 
quantitative parameter of $A D C$ value was included in our risk score because $A D C$ reflects the extent of diffusion hindrance and mobility of water molecules and indicates tumor cellularity and microenvironment [31]. Previously, Suh et al [14] found that an ADC value $\leq 1.11 \times 10^{-3} \mathrm{~mm}^{2} / \mathrm{s}$ was an independent risk factor for predicting MVI with a high sensitivity and specificity of $93.5 \%$ and $72.2 \%$, respectively. Xu et al [15] reported that an $A D C$ value $\leq 1.227 \times 10^{-3} \mathrm{~mm}^{2} / \mathrm{s}\left(b=500 \mathrm{~s} / \mathrm{mm}^{2}\right)$ was an independent risk factor for predicting MVI in $\mathrm{HCC}$ small than $2 \mathrm{~cm}$, with a sensitivity and specificity of $66.7 \%$ and $78.6 \%$, respectively. In our risk score, ADC showed the lowest OR values among the risk factors, the reason may be that $A D C$ values vary widely during measurement and may not be adapted in generalization [32].

Many previous predictive models of MVI may have limited clinical utility because most models are not applicable to the prognostic stratification of early recurrence. Also, several predictive models of early recurrence have been proposed but the relationship between predictive factors of early recurrence and MVI may be lack of interpretation. Our proposed risk score based on MVI status is simple and easily to be evaluated, which may help clinicians to estimate the prognostic stratification of early recurrence after resection with a pathology-based explanation. Patients eligible for surgery with a high risk score of MVI may have a higher rate of early recurrence so that the adjuvant therapy may be needed to be considered.

Our study has limitations. First, due to the small sample size in this retrospective study, selective bias cannot be avoided. Second, some other risk factors of MVI were previously reported but were not included in our analysis. In the limited sample size, we selected some commonly used features that can be easily evaluated to facilitate a clinical use. Third, the risk score was established based on MVI status to provide a prognostic stratification of HCC patients. Some histological factors such as histological grade may be associated with early recurrence and potentially affect our results. Finally, our risk score is preliminary and needs further external validation with use of prospective cohort.

In conclusion, we developed a risk score based on MVI status in HCC by combining the noninvasive radiological and laboratory features to provide a stratification of patients in high risk of early recurrence after resection, which is preliminary and needs further validation.

\section{Abbreviations}

MVI: Microvascular invasion; HCC: hepatocellular carcinoma; AFP: alpha-fetoprotein; PIVKA-II: Prothrombin induced by vitamin K absence-ll; ALT: alanine aminotransferase; AST: aspartate aminotransferase; OR: odds ratio; PPV: positive predictive value; NPV: negative predictive value; ADC: apparent diffusion coefficient; BW: bandwidth; FA: flip angle

\section{Declarations}

Ethics approval and consent to participate: 
All the study protocols were conducted in accordance with the ethical guidelines of the Declaration of Helsinki. This study was approved by the Taizhou first people's Hospital, Fudan University (approval number 2020-KY002-01). The committee waived the requirement for informed consent because it is a retrospective study.

Consent for publication: Not applicable.

Competing interests: no conflict of interest.

Funding: This work was supported by the second batch of social development science and technology plan projects in Taizhou in 2020 (20ywb68)

\section{Authors' contributions:}

All authors have read and approved the manuscript. DJH, GHZ designed and supervised the study; SW, WZZ, ZCZ collected the patient's clinical and MRI data; SW, GHZ analyzed the data; SW drafted the paper.

Acknowledgements: Not applicable

\section{Availability of data and materials:}

The datasets generated during and analyzed during the current study are not publicly available due to protection of study participant privacy but are available from the corresponding author on reasonable request.

\section{References}

1. Bray F, Ferlay J, Soerjomataram I, Siegel RL, Torre LA, Jemal A: Global cancer statistics 2018 : GLOBOCAN estimates of incidence and mortality worldwide for 36 cancers in 185 countries. $C A$ Cancer J Clin 2018, 68(6):394-424.

2. Llovet JM, Zucman-Rossi J, Pikarsky E, Sangro B, Schwartz M, Sherman M, Gores G: Hepatocellular carcinoma. Nat Rev Dis Primers 2016, 2:16018.

3. Imamura H, Matsuyama Y, Tanaka E, Ohkubo T, Hasegawa K, Miyagawa S, Sugawara Y, Minagawa M, Takayama T, Kawasaki S et al: Risk factors contributing to early and late phase intrahepatic recurrence of hepatocellular carcinoma after hepatectomy. J Hepato/ 2003, 38(2):200-207.

4. Park JH, Koh KC, Choi MS, Lee JH, Yoo BC, Paik SW, Rhee JC, Joh JW: Analysis of risk factors associated with early multinodular recurrences after hepatic resection for hepatocellular carcinoma. Am J Surg 2006, 192(1):29-33.

5. Kamiyama T, Nakanishi K, Yokoo H, Kamachi H, Tahara M, Kakisaka T, Tsuruga Y, Todo S, Taketomi A: Analysis of the risk factors for early death due to disease recurrence or progression within 1 year after hepatectomy in patients with hepatocellular carcinoma. World J Surg Onco/2012, 10:107. 
6. Gouw AS, Balabaud C, Kusano H, Todo S, Ichida T, Kojiro M: Markers for microvascular invasion in hepatocellular carcinoma: where do we stand? Liver Transp/2011, 17 Suppl 2:S72-80.

7. Nara S, Shimada K, Sakamoto Y, Esaki M, Kishi Y, Kosuge T, Ojima H: Prognostic impact of marginal resection for patients with solitary hepatocellular carcinoma: evidence from 570 hepatectomies. Surgery 2012, 151(4):526-536.

8. Han J, Li ZL, Xing H, Wu H, Zhu P, Lau WY, Zhou YH, Gu WM, Wang H, Chen TH et al: The impact of resection margin and microvascular invasion on long-term prognosis after curative resection of hepatocellular carcinoma: a multi-institutional study. HPB (Oxford) 2019, 21(8):962-971.

9. Pote N, Cauchy F, Albuquerque M, Voitot H, Belghiti J, Castera L, Puy H, Bedossa P, Paradis V: Performance of PIVKA-II for early hepatocellular carcinoma diagnosis and prediction of microvascular invasion. J Hepatol 2015, 62(4):848-854.

10. Fan LF, Zhao WC, Yang N, Yang GS: Alpha-fetoprotein: the predictor of microvascular invasion in solitary small hepatocellular carcinoma and criterion for anatomic or non-anatomic hepatic resection. Hepatogastroenterology 2013, 60(124):825-836.

11. Svobodova S, Karlikova M, Topolcan O, Pecen L, Pestova M, Kott O, Treska V, Slouka D, Kucera R: PIVKA-II as a Potential New Biomarker for Hepatocellular Carcinoma - A Pilot Study. In Vivo 2018, 32(6):1551-1554.

12. Ma XL, Zhu J, Wu J, Tian L, Gao YY, Zhang CY, Zhou Y, Dai Q, Wang BL, Pan BS et al: Significance of PIVKA-II levels for predicting microvascular invasion and tumor cell proliferation in Chinese patients with hepatitis B virus-associated hepatocellular carcinoma. Oncol Lett 2018, 15(6):8396-8404.

13. Kim H, Park MS, Choi JY, Park YN, Kim MJ, Kim KS, Choi JS, Han KH, Kim E, Kim KW: Can microvessel invasion of hepatocellular carcinoma be predicted by pre-operative MRI? Eur Radiol 2009, 19(7):1744-1751.

14. Suh YJ, Kim MJ, Choi JY, Park MS, Kim KW: Preoperative prediction of the microvascular invasion of hepatocellular carcinoma with diffusion-weighted imaging. Liver Transp/2012, 18(10):1171-1178.

15. Xu P, Zeng M, Liu K, Shan Y, Xu C, Lin J: Microvascular invasion in small hepatocellular carcinoma: is it predictable with preoperative diffusion-weighted imaging? J Gastroenterol Hepatol 2014, 29(2):330-336.

16. Pawlik TM, Delman KA, Vauthey JN, Nagorney DM, Ng IO, Ikai I, Yamaoka Y, Belghiti J, Lauwers GY, Poon RT et al: Tumor size predicts vascular invasion and histologic grade: Implications for selection of surgical treatment for hepatocellular carcinoma. Liver Transp/ 2005, 11(9):1086-1092.

17. Wang WT, Yang L, Yang ZX, Hu XX, Ding Y, Yan X, Fu CX, Grimm R, Zeng MS, Rao SX: Assessment of Microvascular Invasion of Hepatocellular Carcinoma with Diffusion Kurtosis Imaging. Radiology 2018, 286(2):571-580.

18. Ryu T, Takami Y, Wada Y, Tateishi M, Hara T, Yoshitomi M, Momosaki S, Yasumori K, Saitsu H, Okuda K: A Clinical Scoring System for Predicting Microvascular Invasion in Patients with Hepatocellular Carcinoma Within the Milan Criteria. J Gastrointest Surg 2019, 23(4):779-787. 
19. Song D, Wang Y, Wang W, Wang Y, Cai J, Zhu K, Lv M, Gao Q, Zhou J, Fan J et al: Using deep learning to predict microvascular invasion in hepatocellular carcinoma based on dynamic contrast-enhanced MRI combined with clinical parameters. J Cancer Res Clin Oncol2021, 147(12):3757-3767.

20. Price WN: Big data and black-box medical algorithms. Sci Transl Med 2018, 10(471).

21. An C, Kim DW, Park YN, Chung YE, Rhee H, Kim MJ: Single Hepatocellular Carcinoma: Preoperative MR Imaging to Predict Early Recurrence after Curative Resection. Radiology 2015, 276(2):433-443.

22. Kim S, Shin J, Kim DY, Choi GH, Kim MJ, Choi JY: Radiomics on Gadoxetic Acid-Enhanced Magnetic Resonance Imaging for Prediction of Postoperative Early and Late Recurrence of Single Hepatocellular Carcinoma. Clin Cancer Res 2019, 25(13):3847-3855.

23. Shan QY, Hu HT, Feng ST, Peng ZP, Chen SL, Zhou Q, Li X, Xie XY, Lu MD, Wang W et al: CT-based peritumoral radiomics signatures to predict early recurrence in hepatocellular carcinoma after curative tumor resection or ablation. Cancer Imaging 2019, 19(1):11.

24. Zhao Y, Wu J, Zhang Q, Hua Z, Qi W, Wang N, Lin T, Sheng L, Cui D, Liu J et al: Radiomics Analysis Based on Multiparametric MRI for Predicting Early Recurrence in Hepatocellular Carcinoma After Partial Hepatectomy. J Magn Reson Imaging 2021, 53(4):1066-1079.

25. European Association for the Study of the Liver. Electronic address eee, European Association for the Study of the L: EASL Clinical Practice Guidelines: Management of hepatocellular carcinoma. $J$ Hepatol 2018, 69(1):182-236.

26. Chernyak V, Fowler KJ, Kamaya A, Kielar AZ, Elsayes KM, Bashir MR, Kono Y, Do RK, Mitchell DG, Singal AG et al: Liver Imaging Reporting and Data System (LI-RADS) Version 2018: Imaging of Hepatocellular Carcinoma in At-Risk Patients. Radiology 2018, 289(3):816-830.

27. Fujikawa T, Shiraha H, Ueda N, Takaoka N, Nakanishi Y, Matsuo N, Tanaka S, Nishina S, Suzuki M, Takaki A et al: Des-gamma-carboxyl prothrombin-promoted vascular endothelial cell proliferation and migration. J Biol Chem 2007, 282(12):8741-8748.

28. Gao FJ, Cui SX, Chen MH, Cheng YN, Sun LR, Ward SG, Kokudo N, Tang W, Qu XJ: Des-gammacarboxy prothrombin increases the expression of angiogenic factors in human hepatocellular carcinoma cells. Life Sci 2008, 83(23-24):815-820.

29. Zhao WC, Fan LF, Yang N, Zhang HB, Chen BD, Yang GS: Preoperative predictors of microvascular invasion in multinodular hepatocellular carcinoma. Eur J Surg Oncol 2013, 39(8):858-864.

30. Rodriguez-Peralvarez M, Luong TV, Andreana L, Meyer T, Dhillon AP, Burroughs AK: A systematic review of microvascular invasion in hepatocellular carcinoma: diagnostic and prognostic variability. Ann Surg Oncol 2013, 20(1):325-339.

31. Le Bihan D: Apparent diffusion coefficient and beyond: what diffusion MR imaging can tell us about tissue structure. Radiology 2013, 268(2):318-322.

32. Jensen JH, Helpern JA, Ramani A, Lu H, Kaczynski K: Diffusional kurtosis imaging: the quantification of non-gaussian water diffusion by means of magnetic resonance imaging. Magn Reson Med 2005, 53(6):1432-1440. 


\section{Tables}

Table 1: Comparisons of baseline variables between MVI-positive and MVI-negative groups. 


\begin{tabular}{|c|c|c|c|}
\hline Variable & MVI (+) & MVI (-) & $P$ \\
\hline Age (years) & $61 \pm 11$ & $61 \pm 9$ & 0.969 \\
\hline Sex & & & 0.355 \\
\hline Male & 46 & 48 & \\
\hline Female & 5 & 9 & \\
\hline Etiology & & & 0.867 ** \\
\hline Hepatitis B virus & 47 & 53 & \\
\hline Hepatitis $C$ virus & 2 & 3 & \\
\hline None or other & 2 & 1 & \\
\hline Aspartate aminotransferase (IU/L) & $40.5 \pm 30.7$ & $33.5 \pm 33.2$ & 0.259 \\
\hline Alanine aminotransferase (IU/L) & $39.7 \pm 35.0$ & $32.4 \pm 38.9$ & 0.310 \\
\hline Tumor diameter (cm) & $46.9 \pm 28.6$ & $23.3 \pm 10.5$ & $<0.001$ \\
\hline$>2 \mathrm{~cm}$ & 34 & 10 & $<0.001$ \\
\hline$\leq 2 \mathrm{~cm}$ & 17 & 47 & \\
\hline Alpha-fetoprotein (ng/ml) & $742.4(1.5-27738.3)$ & $172.7(1.2-3634.5)$ & $0.093^{*}$ \\
\hline$\geq 20 \mathrm{ng} / \mathrm{ml}$ & 22 & 20 & 0.433 \\
\hline$<20 \mathrm{ng} / \mathrm{ml}$ & 29 & 37 & \\
\hline PIVKA- $\triangle(\mathrm{mAU} / \mathrm{ml})$ & $3526.4(16.7-30000)$ & $412.8(12.1-14817)$ & $<0.001^{*}$ \\
\hline$>32 \mathrm{mAU} / \mathrm{ml}$ & 40 & 18 & $<0.001$ \\
\hline$\leq 32 \mathrm{mAU} / \mathrm{ml}$ & 11 & 39 & \\
\hline Tumor shape & & & 0.001 \\
\hline Irregular & 29 & 15 & \\
\hline Smooth & 22 & 42 & \\
\hline $\operatorname{ADC}\left(\times 10^{-3} \mathrm{~mm}^{2} / \mathrm{sec}\right)$ & $930.7 \pm 102.3$ & $1059.5 \pm 127.4$ & $<0.001$ \\
\hline Capsule enhancement & & & 0.103 \\
\hline Presence & 33 & 28 & \\
\hline Absence & 18 & 29 & \\
\hline
\end{tabular}

* $\mathrm{P}$ value were calculated by using Mann-Whitney $U$ test. 
** Data were compared using the Fisher's exact test.

PIVKA-『: prothrombin induced by vitamin-K-absence-ll;

ADC: apparent diffusion coefficient

Table 2: Univariate and Multivariate analysis of risk scores for MVI in HCC.

\begin{tabular}{|llllll|}
\hline & Univariate & & Multivariate & & \\
& OR $(95 \% \mathrm{Cl})$ & $\mathrm{P}$ & OR $(95 \% \mathrm{Cl})$ & $\mathrm{P}$ & $\begin{array}{l}\text { Risk } \\
\text { score }\end{array}$ \\
\hline Age & & & & & \\
\hline Sex & $0.98(0.95-1.02)$ & 0.337 & & & \\
\hline Hepatitis B virus & $0.13(0.02-1.15)$ & 0.067 & & & \\
\hline Size $>2$ cm & $1.02(0.97-1.04)$ & 0.967 & & 0.024 & 3 \\
\hline Alpha-fetoprotein $\geq 20$ & $9.40(3.83-$ & $<0.001$ & $3.05(1.19-$ & & \\
ng/ml & $1.40(0.65-3.05)$ & 0.392 & & & \\
\hline PIVKA- $>>32$ mAU/ml & $7.88(3.30-$ & $<0.001$ & $4.13(1.23-$ & 0.001 & 4 \\
\hline Irregular Shape & $18.81)$ & & $11.38)$ & & \\
\hline ADC & $3.69(1.64-8.29)$ & 0.002 & $3.10(1.16-8.31)$ & 0.018 & 3 \\
\hline Capsule enhancement & $3.97(1.78-8.85)$ & 0.001 & $2.27(1.14-7.71)$ & 0.035 & 2 \\
\hline Alanine aminotransferase & $0.51(0.36-0.93)$ & 0.034 & $1.90(0.88-4.12)$ & 0.105 & \\
\hline Aspartate aminotransferase & $1.01(0.94-1.02)$ & 0.324 & & & \\
\hline
\end{tabular}

PIVKA-『: prothrombin induced by vitamin-K-absence-II;

ADC: apparent diffusion coefficient

OR: odds ratio; $95 \% \mathrm{Cl}$ : $95 \%$ confidence interval 


\begin{tabular}{|lllll|}
\hline & No. of patients & Early recurrence & No early recurrence & \\
\hline Risk factors & & & & P \\
\hline Size $>2$ cm & 44 & 25 & 19 & 0.048 \\
\hline PIVKA- $>32.01 \mathrm{mAU} / \mathrm{ml}$ & 58 & 33 & 25 & 0.010 \\
\hline Irregular Shape & 44 & 22 & 22 & 0.439 \\
\hline ADC $<$ & 51 & 31 & 20 & 0.002 \\
\hline Risk score* & & & & \\
\hline $8-12$ & 36 & 29 & 7 & $<0.001$ \\
\hline $5-7$ & 23 & 11 & 12 & 0.837 \\
\hline $0-4$ & 49 & 17 & 32 & 0.007 \\
\hline
\end{tabular}

PIVKA-『: prothrombin induced by vitamin-K-absence-II;

ADC: apparent diffusion coefficient

Table 4: Cox survival analysis of MVI-related risk factors and scores for early recurrence of HCC after Resection.

\begin{tabular}{|lllll|}
\hline & Univariate & \multicolumn{3}{l|}{ Multivariate } \\
& HR $(95 \% \mathrm{Cl})$ & $\mathrm{P}$ & $\mathrm{HR}(95 \% \mathrm{Cl})$ & $\mathrm{P}$ \\
\hline Size $>2 \mathrm{~cm}$ & $1.74(1.02-2.96)$ & 0.041 & $1.28(0.38-3.43)$ & 0.684 \\
\hline PIVKA- $>32.01 \mathrm{mAU} / \mathrm{ml}$ & $1.93(1.08-3.46)$ & 0.027 & $1.79(0.91-3.52)$ & 0.090 \\
\hline Irregular Shape & $1.40(0.81-2.41)$ & 0.224 & & \\
\hline ADC & $1.95(1.13-3.36)$ & 0.016 & $1.60(0.65-3.93)$ & 0.302 \\
\hline Risk score & & & & \\
\hline $8-12$ & $2.15(1.26-3.68)$ & 0.001 & $1.86(1.08-3.22)$ & 0.026 \\
\hline $5-7$ & $0.79(0.41-1.55)$ & 0.504 & & \\
\hline $0-4$ & $0.54(0.30-0.96)$ & 0.038 & $1.24(0.45-3.37)$ & 0.679 \\
\hline
\end{tabular}

PIVKA-『: prothrombin induced by vitamin-K-absence-II; 
ADC: apparent diffusion coefficient

HR: Hazard ratio

Table 5: Diagnostic performance of MVI-related risk scores for the prediction of early recurrence after resection of HCC.

\begin{tabular}{|c|c|c|c|c|c|c|}
\hline $\begin{array}{l}\text { Risk } \\
\text { score }\end{array}$ & Risk level & $\begin{array}{l}\text { No. of } \\
\text { patients }\end{array}$ & Sensitivity & Specificity & PPV & NPV \\
\hline $8-12$ & High & 36 & $\begin{array}{l}0.52(0.38- \\
0.65)\end{array}$ & $\begin{array}{l}0.88(0.75- \\
0.95)\end{array}$ & $\begin{array}{l}0.83(0.66- \\
0.93)\end{array}$ & $\begin{array}{l}0.61(0.49- \\
0.72)\end{array}$ \\
\hline $5-7$ & Moderate & 23 & $\begin{array}{l}0.20(0.11- \\
0.33)\end{array}$ & $\begin{array}{l}0.77(0.63- \\
0.87)\end{array}$ & $\begin{array}{l}0.48(0.27- \\
0.69)\end{array}$ & $\begin{array}{l}0.48(0.37- \\
0.59)\end{array}$ \\
\hline $0-4$ & Low & 49 & $\begin{array}{l}0.60(0.46- \\
0.73)\end{array}$ & $\begin{array}{l}0.69(0.55- \\
0.80)\end{array}$ & $\begin{array}{l}0.65(0.50- \\
0.78)\end{array}$ & $\begin{array}{l}0.64(0.51- \\
0.76)\end{array}$ \\
\hline
\end{tabular}

PPV: positive predictive value;

NPV: negative predictive value

\section{Figures}

\section{Figure 1}

Flowchart of excluded patients in our study.

\section{Figure 2}

A 72-year-old patient with the HCC in the left lobe of liver were histologically confirmed of MVI negative status (risk score of 3 points) and did not have early recurrence during postoperative follow-up. (A) Precontrast phase; (B) Arterial phase; (C) Venous phase; (D) high b value map of DWl; (E) ADC map; (F) no $\mathrm{MVI}$ appearance under the microscope.

\section{Figure 3}


A 56-year-old patient with the HCC in the right lobe of liver were histologically confirmed of presence of $\mathrm{MVI}$ (risk score of 12 points) and had early recurrence in 8 months after resection. (A) Pre-contrast phase; (B) Arterial phase; (C) Venous phase; (D) high b value map of DWl; (E) ADC map; (F) presence of MVI under the microscope.

$$
\text { Strata }+ \text { Low risk }+ \text { Moderate risk }+ \text { High risk }
$$
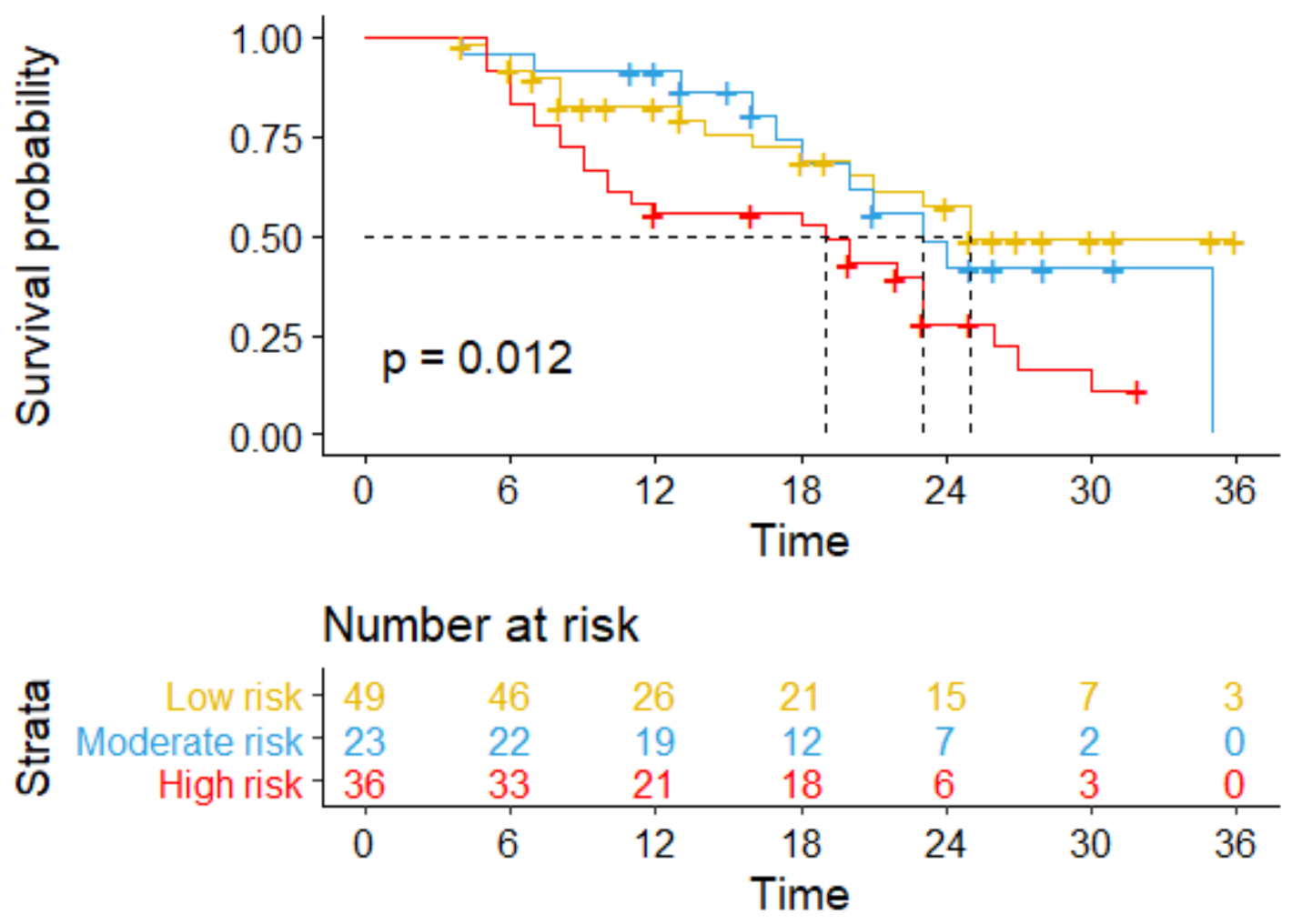

Figure 4

Recurrence-free survival curves (RFS) scaled by risk levels of MVI in the HCC patients. 